\title{
Gene Expression Profile in Liver Transplantation and the Influence of Gene Dysregulation Occurring in Deceased Donor Grafts
}

\author{
Anna Conti ${ }^{1, \dagger}$, Simona Scala ${ }^{2, \dagger}$, Marina Romano ${ }^{2,3}$, Antonella Izzo ${ }^{1}$, Floriana Fabbrini ${ }^{1}$, \\ Floriana Della Ragione ${ }^{4}$, Maurizio D’Esposito ${ }^{4}$, Lucio Nitsch $^{1}$, Fulvio Calise ${ }^{2,3}$ and \\ Antonio Faiella ${ }^{*}, 2$ \\ ${ }^{I}$ Department of Cellular and Molecular Biology and Pathology, Federico II University, Naples, Italy \\ ${ }^{2}$ Center of Biotechnology, “Antonio Cardarelli” Hospital, Padiglione "Y”, Via A. Cardarelli, 9, 80131 Naples, Italy \\ ${ }^{3}$ Liver Transplantation Unit, “Antonio Cardarelli” Hospital, Padiglione "Y”, Via A. Cardarelli, 9, 80131 Naples, Italy \\ ${ }^{4}$ Institute of Genetics and Biophysics, "National Research Council" Naples, Italy
}

\begin{abstract}
Background: Brain dead patients are the main source of organs for transplants. Brain death causes changes in peripheral organs. We define modifications of gene expression in specific pathways occurring in donor livers and their influence on gene expression profile of livers after transplant.

Methods: We compared gene expression profile of both deceased donor livers and transplanted livers to gene expression data of liver tissue, retrieved from Array Express database, used as control. All expression data were obtained by microarray analysis.

Results: The expression of about 33,000 genes has been compared in liver samples from three groups: deceased donor livers, transplanted livers two hours after reperfusion, and control livers. We found that about 900 genes are dysregulated in deceased donor versus control livers. Up-regulated genes are mainly involved in apoptosis, immune response and inflammation. Down-regulated genes are mostly involved in metabolism and electron transport. We also re-evaluated a group of genes that in a previous study were found dysregulated in transplanted livers when compared to donor livers. Most of these genes, but not all, were dysregulated also when compared to control livers. Moreover 317 additional genes, dysregulated after liver transplant, were identified in this study; they were undetectable in the previous study because they had the same dysregulation both in donor and in transplanted livers.

Conclusions: Understanding molecular mechanisms that in the donor compromise graft function is crucial in order to discriminate between basal graft damages and ischemia-reperfusion injuries and therefore to identify therapeutic targets aiming to improve liver transplantation performances.
\end{abstract}

Keywords: Liver transplantation, brain death, gene expression profiling, ischemia/reperfusion injury.

\section{INTRODUCTION}

Organs from brain dead donors are the main source for solid organ transplantation. Brain death (BD) is a complex physiological event, defined as an irreversible injury of cerebrum, cerebellum and brain stem and is associated with severe hemodynamic changes, coagulopathies, pulmonary changes, hypothermia and electrolyte imbalances. Hemodynamic instability associated with brain death can contribute to deterioration of peripheral organs. These changes may predispose the graft to increasing ischemia reperfusion injury (IRI) damages during the transplant process, accelerating organ rejection after transplantation [1]. The hemodynamic instability is well recognized as 'autonomic storm', an initial period of excessive parasympathetic activity, which is immediately followed by a sympathetic activation with high

*Address correspondence to this author at the Center of Biotechnology "Antonio Cardarelli" Hospital, Padiglione "Y", Via A. Cardarelli, 9, 80131 Naples, Italy; Tel: (+39) 3939130 130; Fax: +39 081747 34 33;

E-mail: antonio.faiella@aocardarelli.it

${ }^{\dagger}$ Both authors contributed equally to this work plasma levels of catecholamines, extreme arterial hypertension and tachycardia. During these phases, potential donor grafts undergo a transient period of ischemia [2]. Shock and oxidative injury during the intensive care treatment of the potential donor, followed by brain death, should be regarded as a major risk factor affecting organ viability, post-transplant function and graft survival in organ transplantation, in addition to unavoidable IRI during transplantation procedures. Donor's brain death might cause and promote organ injury altering the immunological and inflammatory status of the graft, increasing both the sensitivity of the organs towards preservation injury and acute rejection following transplantation. Despite this correlation has been experimentally shown, no clinical trials support so far this hypothesis [2].

In a previous study [3] we have compared gene expression levels in transplanted livers, soon after reperfusion, versus basal gene expression levels, before liver retrieval from the donor. About 800 genes were found dysregulated after transplantation, but we have not analyzed the potential effects of brain death on the gene expression variations. 
A very recent study [4] compares gene expression patterns of transplanted livers from living donors with transplanted livers from deceased donors, defining molecular signature of both. The authors focus their attention to the pathways, which show dysregulation in the transplant process both in livers from living donors and from deceased donors; however they do not analyze the basal differences between the grafts from the two groups of donors.

The aim of the present study was to systematically define alterations of gene expression and impairment of specific pathways induced in deceased donor livers when compared to control livers. Based on these alterations an accurate analysis of genes dysregulated in transplanted livers versus control livers was carried out, in order to discriminate between transcriptional changes, due to ischemia reperfusion injuries, and variations possibly caused by brain death and other donor conditions.

We have compared gene expression profile of both deceased donor livers (DL) and transplanted livers (TL) to gene expression data of liver tissue, retrieved from Array Express database, used as control (CL) [3, 5]. This control was a set of livers from sudden death individuals without previous agonal state. Literature data demonstrate that the integrity of mRNA is scarcely affected by sudden death without agonal state. In contrast agonal state preceding death has a substantial effect on gene expression [6,7]. A very large amount of research studies have been conducted on deceased tissues and also information included in all the databases reporting normal gene expression throughout the tissues [8] is derived from autoptic studies.

The expression of about 900 genes was found dysregulated in DL if compared to controls. This wide gene expression modification clearly affects gene regulation in TL.

The data reported in the present study give new insight to clarify the consequences of brain death and intensive care injuries on the human orthotopic liver transplantation by a molecular point of view, and help us to recognize new therapeutic targets useful to improve orthotopic liver transplantation (OLT) performance.

\section{MATERIALS AND METHODOLOGY}

\section{Experimental Design}

Gene expression profile of samples from DL and TL was compared to gene expression profile of samples from CL. The choice of sample size was performed by running pvalues of expression data comparisons among groups through the PowerAtlas software $[9,10]$. We selected a sample size of ' 5 ' per group to obtain an Expected Discovery Rate (EDR) $>40 \%(63 \%)$ and a True Positive Probability (TP) $>80 \%$ (98\%) (Supplementary file $\mathbf{1}$ ).

Then, five biological replicates per condition were analyzed. All expression data (DL, TL and CL) were obtained by microarray analysis, using Affymetrix gene chip HGU133 Plus 2. Hybridization data were normalized and quantified using Robust Multiarray Analysis (RMA) software [11]. DL and TL samples were collected as previously described [3]. Briefly, 10 liver biopsies were analyzed: 5 biopsies, from the donors, collected before explantation (DL group); 5 biopsies from the transplant recipients (TL group), collected 2-3 hours after liver reperfusion. Donors were classified as 'standard' according to the criteria of the 'Italian National Transplantation Center'. Particularly, the donors' age ranged from 38 to 83 years, no hypotension, steatosis always less than $15 \%$. Mean cold ischemia time was 8 hours (ranging from seven to ten hours), mean warm ischemia $45 \mathrm{~min}$, mean hospitalization in intensive care unit was 5 days [3]. Expression data of livers, from five healthy individuals who suffered sudden death, were retrieved from Array Express database (SAMPLE ID: E-AFMX-11) [5, 12] and used as control samples. All individuals, 3 males and 2 females, suffered sudden death for reason other than their participation to the study and without any relation to the tissues used. Age was ranging from 27 to 29 and was unknown in 2 cases. Total RNAs, isolated from $200 \mathrm{mg}$ of frozen tissues using the Trizol reagent, were of high and comparable quality as gauged by the ratio of $28 \mathrm{~S}$ to $18 \mathrm{~S}$ ribosomal RNAs estimated using the Agilent 2100 Bioanalyzer (range 1.4-1.6) [12].

In order to validate the use of deceased tissues as controls, we compared our results to a set of expression data from microarray analysis (same technology and experimental conditions) of six liver biopsies from living donors, available very recently from GEO repository $[4,13]$ (Supplementary file 2).

\section{RNA Extraction, Data Acquisition and Analysis}

DL and TL samples were treated as previously described [3]. Array data have been deposited in GEO data base with accession number GSE14951 [14]. Expression data were pre-filtered to reduce noise and discard 'unreliable' genes using the Cross-Gene error model [14]. The analysis of functional clusters was performed on lists of differentially expressed genes for both Gene Ontology (GO) categories and biological pathways. GO functional class scoring was performed using the web-based GOTM software $[15,16]$ which visualizes differentially expressed genes in the GO context, considering as gene sets all the GO categories for biological processes, molecular functions and cellular components. The list of differentially expressed genes was compared to the complete list of genes spotted on Affymetrix HG-U133 Plus 2 chip, in order to identify GO categories of genes significantly $(\mathrm{p}<0.01)$ more represented in the list of differentially expressed genes than in the reference gene set.

\section{Real-Time Quantitative PCR}

Expression values of 26 genes chosen among the most dysregulated genes were checked by RT-PCR. The same batch of total mRNA was used for both microarray and validation experiments in DL and TL. RNAs, from five liver tissue obtained during resection of benign focal lesions, were used as controls in RT-PCR experiments. Two biopsies came from liver resections of two females (24 and 26 aged) suffering by hepatocellular adenoma. Two biopsies came from liver resections of giant hepatic cystis from two male aged respectively 39 and 43 . The last biopsy came from a liver of a male suffering by liver hemangioma. cDNA was synthesized Real Time PCR were performed as previously described [3]. PCR reactions were performed in triplicate. The primers (Primm Biotech Products and Services, Milan - 
Table 1. Primer Pairs Used for Real-Time Quantitative PCR

\begin{tabular}{|c|c|c|c|c|}
\hline Probe Set & Gene Name & Ensembl Gene ID & Left Primer & Right Primer \\
\hline 205364_at & ACOX 2 & ENSG00000168306 & CGGAGTCTTCAGGACCACAC & GCAGGAAGCCATTGTCTGTT \\
\hline 209186_at & ATP2A2 & ENSG00000174437 & TGAAACAGTTCATCCGCTACC & AATCAAAGCCTCGGGAAATC \\
\hline 228876_at & BAIAP2L2 & ENSG00000128298 & AGAACGTGCGGGAGATGAAG & CAGACACGAAGGCCTGCAT \\
\hline 204093_at & $\mathrm{CCNH}$ & ENST00000256897 & CAGAAGTTGGAGCGATGTCA & GTCCATTCTTCCTCCTCATGTT \\
\hline 220046_s_at & CCNL1 & ENSG00000163660 & GAACTCCAGCCCTTTCAACC & TTGGTGATTTCTCTTCAGCTTTT \\
\hline 228766_at & CD36 & ENSG00000135218 & GCTGAGGACAACACAGTCTCTTTC & AGCCTCTGTTCCAACTGATAGTGA \\
\hline 213279_at & DHRS1 & ENSG00000157379 & TGGCCGTGGCATTGC & AGATGGCGGCCAGTGATG \\
\hline 241945_at & HECTD1 & ENSG00000092148 & ACTAATGCCACGAACAACATGAAT & TGATGTAGTACCAGGTGTGGTCAA \\
\hline 201466_s_at & JUN & ENSG00000177606 & AGAAAGTCATGAACCACGTTAACAGT & CCCCCGACGGTCTCTCTT \\
\hline 205222_at & LBP & ENSG00000129988 & CCGACTGACCACCAAGTCCTT & GGCACTGATCCCTGGAGTTC \\
\hline 203675_at & NUCB2 & ENSG00000070081 & GAAAAGGCAAGAAGTAGGAAGGTT & GTCAGGATTCAGGTGGTTTAGG \\
\hline 206278_at & PTAFR & ENSG00000169403 & CCTGCCACTTTGGATTGTCTACT & GCCACGTTGCACAGGAATTT \\
\hline 210479_s_at & RORA & ENSG00000069667 & TCATTCTCCACCCAGCTGTTG & CTGTGCTTTGCCCCAGTGTA \\
\hline 222226_at & SAA3P & ENSG00000166787 & GCCAGGCTACCAACAAATGG & GCAGATTGAAAAGGAAGCTCAGTAT \\
\hline 213874_at & SERPINA4 & ENSG00000100665 & TCAAAGCCCTGTGGGAGAAA & CGGACTGTTGTGTTCTCATCAAC \\
\hline 239818_x_at & TRIB1 & ENSG00000173334 & GGGCGCTGTGCATCCA & AAGGCCTGATTTTGTCCTGGTA \\
\hline
\end{tabular}

Italy) used for amplification are listed in Table 1. Primer pairs were designed using the Primer 3 software [17] in order to obtain amplicons ranging from 100 and $150 \mathrm{bp}$, and specifically designed to span introns or cross intron/exon boundaries. Data normalization was performed using GAPDH as housekeeping gene [18]. Experiments were performed twice, in triplicate. The amplification protocol was: 1 cycle of $10 \mathrm{~min}$ at $95{ }^{\circ} \mathrm{C}, 40$ cycles of $95{ }^{\circ} \mathrm{C}$ for 15 sec, $58-60{ }^{\circ} \mathrm{C}$ for $20 \mathrm{sec}, 72{ }^{\circ} \mathrm{C}$ for $20 \mathrm{sec}$, plus an extension at $72{ }^{\circ} \mathrm{C}$ for $3 \mathrm{~min}$. The relative expression value was calculated with the formula $2^{\text {DDct }}$

\section{Statistics}

Expression data from different groups were compared using the ANOVA test, with Benjamini and Hochberg false discovery rate as multiple testing corrections. Statistical significance was established at $\mathrm{p}<0.01$. Genes were considered differentially expressed with a fold change $>1.5$ between two conditions. Gene sets were considered enriched with a $\mathrm{p}$ $<0.01$ when compared to the reference gene list.

Liver biopsies were collected in different hospitals, including the "Liver Transplantation Center" of the "Cardarelli Hospital". All biopsies were obtained with informed consent given according to protocols approved by the Institutional Ethics Committee of the "Antonio Cardarelli Hospital."

\section{RESULTS}

The expression of about 33,000 genes, represented on the Affymetrix chip HG-U133 Plus 2, has been evaluated in liver samples from three groups: DL, TL 2 hours after reperfusion, and CL. The groups were compared each other for gene expression. Expression of about 900 genes was dysregulated in DL compared to control ones. Table $\mathbf{2}$ shows the 40 most up-regulated and the 40 most down-regulated genes. Table $\mathbf{3 a}$ and Table $\mathbf{3} \mathbf{b}$ report the distribution of the dys- 
Table 2. The Most up- and Down-Regulated Genes in DL Group vs. CL Group

\begin{tabular}{|c|c|c|c|}
\hline \multicolumn{2}{|c|}{ Up-Regulated Genes } & \multicolumn{2}{|c|}{ Down-Regulated Genes } \\
\hline Gene Name & Fold Change & Gene Name & Fold Change \\
\hline SPINK1 & 95.29 & LOC 283130 & 0.0555 \\
\hline APOC3 & 9.516 & MALAT-1 & 0.0612 \\
\hline RPS11 & 8.807 & $\mathrm{TF}$ & 0.084 \\
\hline LOC440836 & 6.052 & H19 & 0.112 \\
\hline ATP2A2 & 5.483 & CAT & 0.116 \\
\hline APOA1 & 5.461 & GNMT & 0.117 \\
\hline TOMM40 & 5.194 & G6PC & 0.13 \\
\hline APOC2 & 4.937 & C8orf4 & 0.139 \\
\hline TMEM151 & 4.83 & SLC6A1 & 0.145 \\
\hline RBP4 & 4.765 & HECTD1 & 0.146 \\
\hline C9ORF44 & 4.72 & KIAA0293 & 0.155 \\
\hline FAM84A & 4.72 & C6orf71 & 0.159 \\
\hline SLC39A8 & 4.689 & GPLD1 & 0.164 \\
\hline LTB4R2 & 4.59 & TRIB1 & 0.168 \\
\hline RP3-402G11.12 & 4.375 & DKFZP586A0522 & 0.176 \\
\hline TGM2 & 4.062 & C10orf65 & 0.202 \\
\hline BAIAP2L2 & 4.039 & SLC25A15 & 0.203 \\
\hline SOX15 & 3.996 & JUN & 0.204 \\
\hline DLG4 & 3.995 & RCL1 & 0.204 \\
\hline SLC35C1 & 3.919 & ZGPAT & 0.204 \\
\hline ELMOD2 & 3.911 & DSIPI & 0.207 \\
\hline DDX54 & 3.9 & KLF6 & 0.209 \\
\hline AMBP & 3.876 & CYP26A1 & 0.21 \\
\hline SCUBE1 & 3.874 & NDUFS1 & 0.213 \\
\hline SFXN4 & 3.865 & AASS & 0.218 \\
\hline TAOK2 & 3.782 & CYP4A11 & 0.218 \\
\hline VWA1 & 3.779 & TMEM16A & 0.22 \\
\hline SRCRB4D & 3.744 & BAAT & 0.221 \\
\hline ATF5 & 3.709 & HGD & 0.221 \\
\hline ВBC3 & 3.678 & PSMAL & 0.232 \\
\hline
\end{tabular}


Table 3a. Enriched GO Categories of Genes Up-Regulated in Donor Livers versus Controls

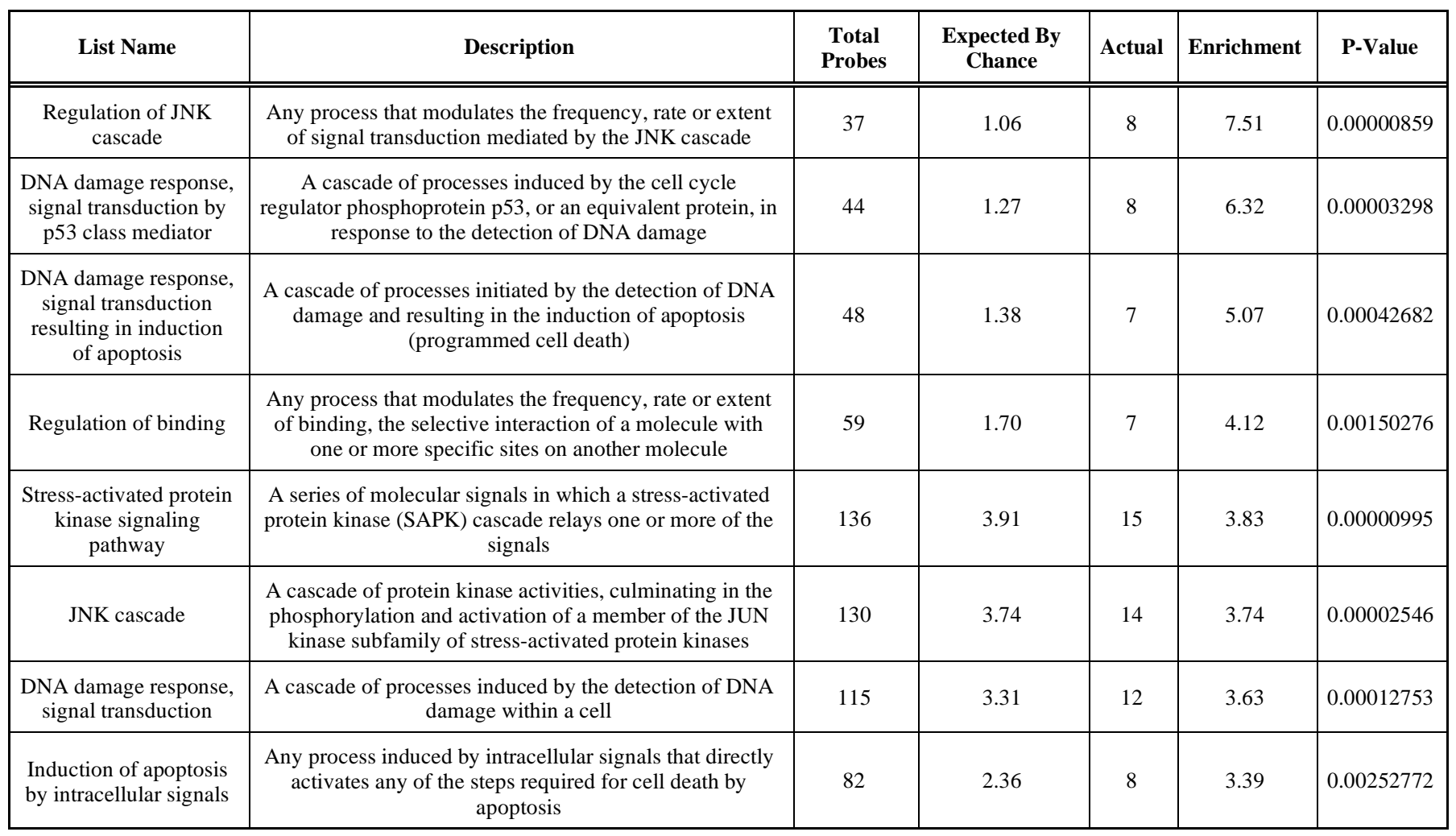

Table 3b. Enriched GO Categories of Genes Down-Regulated in Donor Livers versus Controls

\begin{tabular}{|c|c|c|c|c|c|c|}
\hline List Name & Description & $\begin{array}{l}\text { Total } \\
\text { Probes }\end{array}$ & $\begin{array}{l}\text { Expected } \\
\text { by Chance }\end{array}$ & Actual & Enrichment & p-Value \\
\hline $\begin{array}{l}\text { Heterocycle metabolic } \\
\text { process }\end{array}$ & $\begin{array}{l}\text { The chemical reactions and pathways involving } \\
\text { heterocyclic compounds, those with a cyclic } \\
\text { molecular structure and at least two different atoms in } \\
\text { the ring (or rings) }\end{array}$ & 146 & 3.93 & 17 & 4.33 & 0.00000050 \\
\hline $\begin{array}{l}\text { Monocarboxylic acid } \\
\text { metabolic process }\end{array}$ & $\begin{array}{l}\text { The chemical reactions and pathways involving } \\
\text { monocarboxylic acids, any organic acid containing } \\
\text { one carboxyl (COOH) group or anion (COO-) }\end{array}$ & 396 & 10.65 & 41 & 3.85 & 0.00000000 \\
\hline Fatty acid metabolic process & $\begin{array}{c}\text { The chemical reactions and pathways involving fatty } \\
\text { acids, aliphatic monocarboxylic acids liberated from } \\
\text { naturally occurring fats and oils by hydrolysis }\end{array}$ & 283 & 7.61 & 29 & 3.81 & 0.00000000 \\
\hline $\begin{array}{l}\text { Aromatic compound } \\
\text { metabolic process }\end{array}$ & $\begin{array}{l}\text { The chemical reactions and pathways involving } \\
\text { aromatic compounds, any organic compound } \\
\text { characterized by one or more planar rings, each of } \\
\text { which contains conjugated double bonds and } \\
\text { delocalized pi electrons }\end{array}$ & 211 & 5.68 & 20 & 3.52 & 0.00000142 \\
\hline Sterol metabolic process & $\begin{array}{l}\text { The chemical reactions and pathways involving } \\
\text { sterols, steroids with one or more hydroxyl groups } \\
\text { and a hydrocarbon side-chain in the molecule }\end{array}$ & 147 & 3.95 & 13 & 3.29 & 0.00018779 \\
\hline Electron transport & $\begin{array}{l}\text { The transport of electrons from an electron donor to } \\
\text { an electron acceptor }\end{array}$ & 599 & 16.11 & 48 & 2.98 & 0.00000000 \\
\hline Alcohol metabolic process & $\begin{array}{c}\text { The chemical reactions and pathways involving } \\
\text { alcohols, any of a class of alkyl compounds } \\
\text { containing a hydroxyl group }\end{array}$ & 541 & 14.55 & 39 & 2.68 & 0.00000005 \\
\hline Glucose metabolic process & $\begin{array}{l}\text { The chemical reactions and pathways involving } \\
\text { glucose, the aldohexose gluco-hexose, D-glucose is } \\
\text { dextrorotatory and is sometimes known as dextrose }\end{array}$ & 195 & 5.25 & 14 & 2.67 & 0.00090344 \\
\hline
\end{tabular}


regulated genes in GO biological process categories, showing that apoptosis and stress activated protein kinase activities are the most affected pathways by up-regulated genes (Table 3a), whereas down-regulated genes are involved in metabolic pathways and electron transport (Table $\mathbf{3 b}$ ). At least 30 mitochondrial enzymes, involved in oxidative chain, are from 2 to 5 folds down-regulated, in deceased DL, if compared to CL (Table 4). Comparison between TL and CL samples demonstrated that 855 genes were dysregulated in TL versus CL. Condition tree from hierarchical clustering of these genes (Fig. 1) clearly shows that about $1 / 3$ of these genes were already dysregulated in DL if compared to controls: 182 genes, (mainly involved in oxidoreductase, electron transport and metabolic activity), were already down-regulated in DL group versus CL group and 135 genes, (involved in inflammatory pathways and cell adhesion), were already up-regulated in DL group versus CL group.

In conclusion, the present study demonstrated that: 1) 900 genes were dysregulated in DL if compared to controls; 2) 855 genes were dysregulated in TL if compared to CL. At least 400 of these genes were already dysregulated in DL.

A comparison of expression data between biopsies of living donors and livers from sudden death individuals is shown in the supplemental section (Supplementary file 2).

Briefly, even though 217 genes are differentially expressed with fold change $>1.75$ and $p<0.01$ between livers

Table 4. Oxidative Phosphorilation Genes Down-Regulated in Donor Livers versus Controls

\begin{tabular}{|c|c|c|c|}
\hline Gene Name & Gene Bank & Description & Fold Change DL vs. CL \\
\hline AASS & AK023446 & aminoadipate-semialdehyde synthase & 0.218 \\
\hline ACOX3 & BF055171 & acyl-Coenzyme A oxidase 3, pristanoyl & 0.534 \\
\hline ALDH5A1 & NM_001080 & aldehyde dehydrogenase 5 family, member A1 & 0.336 \\
\hline $\mathrm{CHDH}$ & AA609488 & choline dehydrogenase & 0.493 \\
\hline COX15 & AF026850 & COX15 homolog, cytochrome c oxidase assembly protein (yeast) & 0.358 \\
\hline $\mathrm{COX} 7 \mathrm{~A} 2 \mathrm{~L}$ & NM_004718 & cytochrome c oxidase subunit VIIa polypeptide 2 like & 0.547 \\
\hline CYP26A1 & NM_000783 & cytochrome P450, family 26 , subfamily A, polypeptide 1 & 0.21 \\
\hline CYP3A5 & AW964006 & cytochrome P450, family 3 , subfamily A, polypeptide 5 & 0.19 \\
\hline CYP3A7 & AF315325 & cytochrome P450, family 3 , subfamily A, polypeptide 7 & 0.297 \\
\hline CYP4A11 & ВC041158 & cytochrome P450, family 4, subfamily A, polypeptide 11 & 0.218 \\
\hline DAO & NM_001917 & D-amino-acid oxidase & 0.499 \\
\hline FLJ22378 & NM_025078 & hypothetical protein FLJ22378 & 0.524 \\
\hline $\mathrm{HAO} 2$ & NM_016527 & hydroxyacid oxidase 2 (long chain) & 0.322 \\
\hline IBRDC2 & AI953847 & IBR domain containing 2 & 0.502 \\
\hline IVD & NM_002225 & isovaleryl Coenzyme A dehydrogenase & 0.488 \\
\hline NISCH & NM_007184 & nischarin & 0.549 \\
\hline PAOX & AI743990 & polyamine oxidase (exo-N4-amino) & 0.523 \\
\hline PIPOX & AF136970 & pipecolic acid oxidase & 0.437 \\
\hline UQCRB & BC005230 & ubiquinol-cytochrome $\mathrm{c}$ reductase binding protein & 0.437 \\
\hline ZDHHC4 & NM_018106 & zinc finger, DHHC domain containing 4 & 0.556 \\
\hline
\end{tabular}




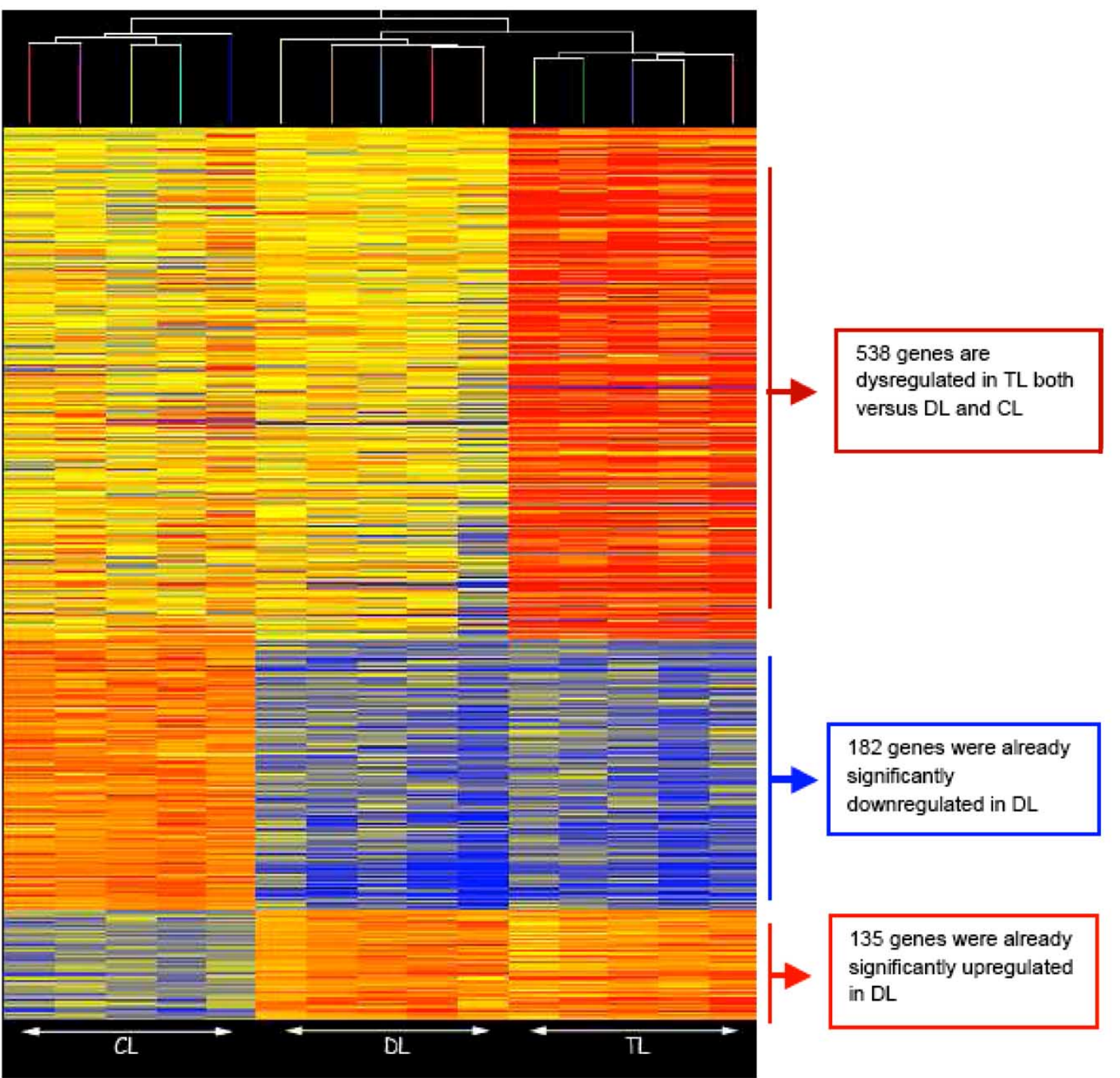

Fig. (1). Condition tree from hierarchical clustering of genes differentially expressed between TL and CL groups.

Samples from each group are well clustered together according to the expression level of these genes. The figure shows that many genes (in the lower part of the graphic), differentially expressed between TL and CL, were already dysregulated in the DL. Therefore their dysregulation might be due to brain death more than to ischemia/reperfusion injury. The genes in the upper part of the tree are dysregulated only in TL group both versus CL and DL samples.

Genes were considered differentially expressed with $\mathrm{p}<0.01$ using ANOVA test with Benjamini and Hochberg false discovery rate correction.

$\mathrm{CL}=$ Control livers, $\mathrm{DL}=$ Donor livers, $\mathrm{TL}=$ Transplanted livers.

from living donors and livers from deceased subjects (CL) only 54 (63 probe sets) out of these genes are included in the set of 900 genes we found differentially expressed in DL vs. CL.

Quantitative RT-PCR analysis of 26 genes, chosen among the most dysregulated genes, was carried out in order to validate the results of microarray analysis. The RT-PCR experiments confirm the microarray data for these genes. Correlation between quantitative RT-PCR and microarray data was satisfactory for all the tested genes $(r>0.85)$ (Table 5).

\section{DISCUSSION}

Clinical studies on humans have shown that allograft from unrelated living donors have better graft function and survival than allograft from deceased donors [19-21]. This difference could be attributed to the pathophysiological changes derived from brain death and donor condition more than to the influence of cold ischemia times [22-24].

In this study we have demonstrated that brain death, together with other factors related to donor condition (shock, intensive care treatment, parenteral nutrition, etc.) causes the dysregulation of at least 900 genes in human liver tissue. The 
Table 5. Comparison between Quantitative Real Time PCR and Microarray Data

\begin{tabular}{|c|c|c|c|}
\hline Probe Set & Gene Name & Microarray Fold Change DL/CL & RT Fold Change DL/CL \\
\hline 205364_at & ACOX2 acyl-Coenzyme A oxidase 2 & 0.27 & 0.27 \\
\hline 209186_at & ATP2A2 ATPase, $\mathrm{Ca}++$ transporting & 5.48 & 1.90 \\
\hline 228876_at & BAIAP2L2 BAI1-associated protein 2-like 2 & 4.04 & 3.50 \\
\hline 204093_at & CCNH cyclin $\mathrm{H}$ & 1.06 & 0.90 \\
\hline 220046_s_at & CCNL1 cyclin L1 & 0.47 & 0.30 \\
\hline 228766_at & CD36 CD36 molecule & 0.13 & 0.25 \\
\hline 213279_at & DHRS1 dehydrogenase/reductase & 0.20 & 0.28 \\
\hline 241945_at & HECTD1 HECT domain-containing 1 & 0.15 & 0.34 \\
\hline 201466_s_at & JUN jun oncogene & 0.20 & 0.18 \\
\hline 205222_at & LBP lipopolysaccharide binding protein & 4.08 & 6.55 \\
\hline 203675_at & NUCB2 nucleobindin 2 & 3.95 & 3.90 \\
\hline 206278_at & PTAFR platelet-activating factor receptor & 4.32 & 7.80 \\
\hline 210479_s_at & RORA RAR-related orphan receptor A & 0.14 & 0.33 \\
\hline 222226_at & SAA3P serum amyloid A3 pseudogene & 4.07 & 6.05 \\
\hline 213874_at & SERPINA4 serpin peptidase inhibitor & 0.19 & 0.20 \\
\hline 239818_x_at & TRIB1 Tribless homolog 1 (Drosophila m.) & 0.17 & 0.10 \\
\hline
\end{tabular}

validation study (Supplementary file 2) demonstrates that 54 of them $(6 / \%)$ might be affected by the choice of deceased tissues, as they are already dysregulated between CL and living donor tissues. Subtraction of these genes from the set of 900 genes does not affect at all the following considerations about injuries occurring in donor livers. Up-regulated genes are mainly involved in immune response, cytoskeletal remodelling, inflammation, apoptosis and cell adhesion. Down-regulated genes are mostly involved in mitochondrial activities and metabolism, being members of metabolic pathways of aminoacids, such as Gly, Ser and Cys, fatty acids (HNF4alpha) and vitamins. Metabolism might be also affected by starvation and stress, due to a long stay in intensive care unit. Molecular and cellular alterations triggered by brain death itself may significantly alter both early and longterm results of transplantation if compared to organs harvested from living donors [25].

Apoptosis induction and increased expression of apoptosis related proteins were observed in hepatocytes from brain dead animals [26]. A recent study on molecular changes induced in the heart by brain death [27], evaluates the variation in the expression levels of 5 genes involved in apoptotic processes: $B A X, B C L 2, C A S P 3$, Cytochrome $\mathrm{C}$ ( $C Y C S)$ and $F A S$ and one gene induced by hypoxia (HIFIA) concluding that brain death mainly induces the expression of 3 out of these genes: $B A X, F A S$ and $C A S P 3$ involved in apoptosis activation. HIFIA is not significantly induced, excluding hypoxic damages. Our results show that $B A X$ and $F A S$ are induced in liver samples from deceased donors. Moreover in our experiments the anti-apoptotic $B C L 2$ gene is induced much more than in the heart as like as HIF1A, indicating that hypoxic injuries are already present in DL.

Our study unraveled that in deceased DL at least 30 mitochondrial enzymes, involved in oxidative chain, are from 2 to 5 fold down-regulated if compared to CL (Table 4). It is known that during ischemia oxidative phosphorylation and ATP level decrease producing ischemic damage. The 


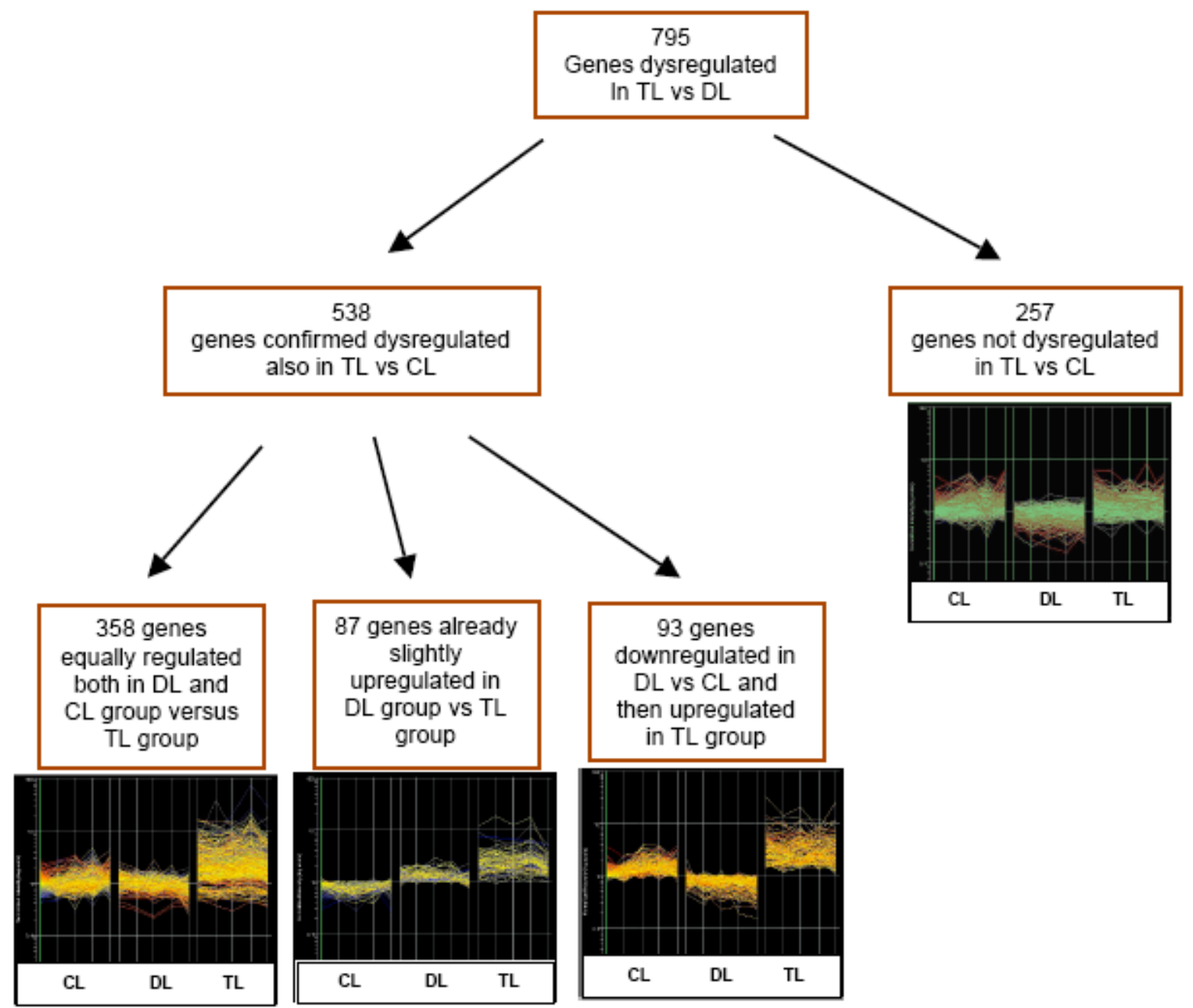

Fig. (2). Expression profiles of 795 genes previously found dysregulated in livers after transplant.

The figure shows on the left the expression profiles of 538 genes found dysregulated in a previous study, in TL versus DL, and now confirmed as dysregulated in TL if compared to CL. Detailed analysis of these genes, dysregulated both versus DL and CL, shows three different behaviors:

- 358 genes were normoregulated between DL and CL groups;

- 87 genes were already slightly up-regulated in DL group.

- 93 genes were down-regulated in DL group versus CL group and highly up-regulated after transplantation in TL group.

On the right side of the figure are shown expression profiles of 257 genes dysregulated in TL versus DL, but not confirmed as dysregulated in TL when compared to CL.

Genes were considered differentially expressed with $\mathrm{p}<0.01$ using ANOVA test with Benjamini and Hochberg false discovery rate correction.

$\mathrm{CL}=$ Control livers, $\mathrm{DL}=$ Donor livers, $\mathrm{TL}=$ Transplanted livers.

situation might be deeply compromised if the expression of oxidative enzymes is down-regulated.

A detailed analysis of 795 genes previously found dysregulated in TL when compared to DL [3], in the new perspective of the comparison between transplanted and control liver tissues, shows that about 250 genes, mainly involved in angiogenesis, lipid metabolism, growth, cell cycle, were not confirmed as dysregulated in our study when compared to CL (Fig. 2). For these genes we hypothesize that their dysregulation is due to events related to brain death instead of IRI. However many genes reported as dysregulated in the previous study are dysregulated even when TL are compared to CL: 87 of them were already slightly up-regulated in DL group. These genes are mainly apoptotic regulators, proteolytic enzymes, chemokines, cytokines and stress responsive genes. Ninetythree more genes, down-regulated in DL, are essentially involved in growth, angiogenesis, mitosis initiation, cell cycle regulation and metabolism. We demonstrate that $\mathrm{c}-F O S$ and $c-J U N$ are down-regulated by brain death causing inhibition of cell proliferation and $V E G F$ signaling (angiogenesis). This might explain the better graft function and survival of allograft from living donors if compared to 
Table 6. 15 Out of 78 Genes Predictive for IPGF, Already Dysregulated in Donor Livers versus Controls

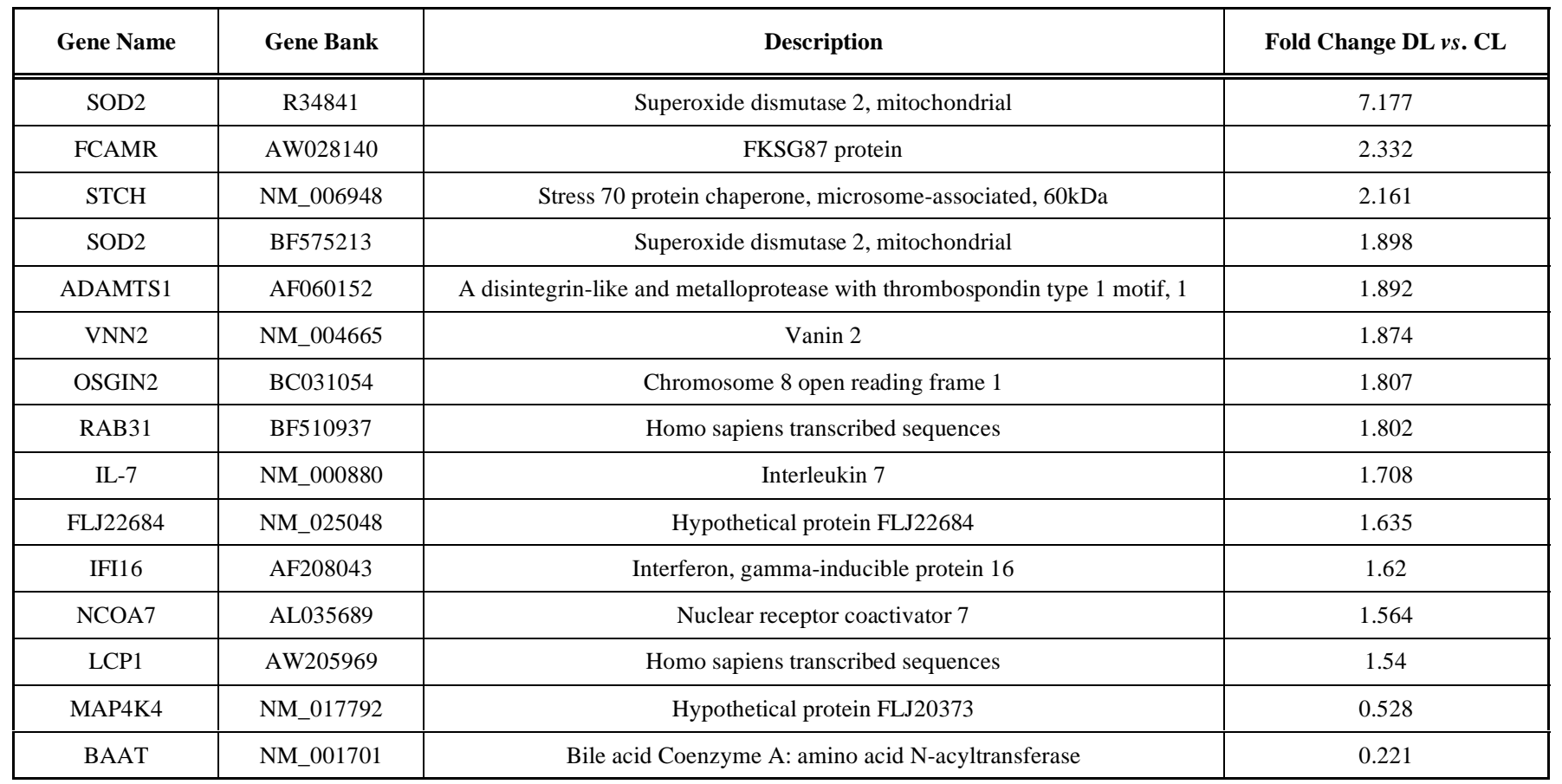

deceased donors. All these genes are very highly up-regulated after transplantation and reperfusion.

In addition to the genes already found dysregulated in the previous study and confirmed in this study, we found 317 additional genes differentially expressed between TL and CL (Fig. 1); these genes were not detected in our previous study [3] because they were already dysregulated in DL, therefore no differences between DL and TL group were detectable. In detail, 135 genes were equally up-regulated in DL and TL group versus controls. They are mainly anti-apoptotic genes, $N F K B$ subunits, $B C L 2, B C L X L$, cell adhesion molecules. 182 genes were equally down-regulated in DL and TL group versus controls. Most of them are involved in fatty acid, glucose and aminoacid metabolism and electron transport activities. Thus, inflammatory processes and oxidative phosphorylation activities are impaired in the liver since the pre-explant period, and continue to be impaired after transplant and reperfusion.

Functional analysis of the remaining genes dysregulated in TL group versus CL group completely confirms the results of our previous study [3]. Many authors agree with the idea that understanding molecular bases of graft failure is crucial to identify therapeutic targets able to improve transplant performance [28]. In this respect, a recent study identifies 78 classifier genes whose dysregulation after liver transplantation is able to predict initial poor graft function (IPGF) [29]. Our study demonstrated that 15 of these genes resulted already dysregulated in DL (Table 6); molecular pathways involved in IPGF might be compromised before the organ is explanted and preventing these mechanisms in the donors possibly results in better graft function.

Recently De Jonge et al. [4] have been carried out in human OLT a study which analyzes the differential gene expression between donor baseline biopsies and postreperfusion biopsies in two groups of liver transplantations: from living donors and from deceased donors. The authors find a large number of genes differentially expressed in both graft types following reperfusion when compared to the pre biopsies, more in the living transplant than in the deceased one. Among the group of genes differentially expressed in post-reperfusion biopsies of both groups they find a significant up-regulation of genes involved in inflammatory and immune processes, both in deceased and in living donor grafts.

\section{CONCLUSIONS}

Brain death and intensive care injuries induce stress in DL, affecting liver gene transcriptional profile both in donors and in recipients, and many genes dysregulated in TL versus CL are already dysregulated in DL before transplantation. On these bases we hypothesize that the dysregulation of these genes, mainly involved in inflammatory pathways, cell adhesion and electron transport, might affect graft function and organ survival in OLT. The insight of these mechanisms is crucial for the identification of therapeutic targets, aiming to improve OLT performances.

\section{ACKNOWLEDGEMENT}

The authors thank R.A. Chamuleau (Academic Medical Center, University of Amsterdam, The Netherlands) for helpful comments and suggestions and A. Ceriello and V. Scuderi (Liver Transplantation Unit, "Antonio Cardarelli" Hospital - Naples, Italy) for their precious surgical assistance. This work has been supported by grants from "Alto Comitato per i Trapianti Regione Campania". 


\section{ABBREVIATIONS}

$$
\begin{aligned}
& \mathrm{BD}=\text { Brain death } \\
& \mathrm{CL}=\text { Control Livers } \\
& \mathrm{DL}=\text { Donor livers } \\
& \mathrm{EDR}=\text { Expected Discovery Rate } \\
& \mathrm{GO}=\text { Gene Ontology } \\
& \mathrm{IRI}=\text { Ischemia Reperfusion Injury } \\
& \mathrm{OLT}=\text { Orthotopic Liver Transplantation } \\
& \mathrm{RMA}=\text { Robust Multiarray Analysis } \\
& \mathrm{TL}=\text { Transplanted Livers }
\end{aligned}
$$

\section{SUPPLEMENTARY MATERIAL}

Supplementary material is available on the publishers Web site along with the published article.

\section{REFERENCES}

[1] Colombo G, Gatti S, Turcatti F, et al. Alteration in the transcriptional profile of livers from brain-dead organ donors. Transplantation 2006; 82: 69-79.

[2] Weiss S, Kotsch K, Francuski M, et al. Brain death activates donor organs and is associated with a worse $\mathrm{I} / \mathrm{R}$ injury after liver transplantation. Am J Transplant 2007; 7: 1584-93.

[3] Conti A, Scala S, D'Agostino P, et al. Wide gene expression profiling of ischemia-reperfusion injury in human liver transplantation. Liver Transpl 2007; 13: 99-113.

[4] de Jonge J, Kurian S, Shaked A, et al. Unique early gene expression patterns in human adult-to-adult living donor liver grafts compared to deceased donor grafts. Am J Transplant 2009; 9: 75872.

[5] Parkinson H, Kapushesky M, Kolesnikov N, et al. ArrayExpress update-from an archive of functional genomics experiments to the atlas of gene expression. Nucl Acids Res 2009; 37: 868-72.

[6] Franz H, Ulmann C, Becker A, et al. Systematic analysis of gene expression in human brains before and after death. Genome Biol 2005; 6: R112.

[7] Li JZ, Vawter MP, Walsh DM, et al. Systematic changes in gene expression in postmortem human brains associated with tissue $\mathrm{pH}$ and terminal medical conditions. Hum Mol Genet 2004; 13: 60916.

[8] Wu C, Orozco C, Boyer J, et al. BioGPS: an extensible and customizable portal for querying and organizing gene annotation resources. Genome Biol 2009; 10: RI30.

[9] Gadbury GL, Garrett KA, Allison DB. Challenges and approaches to statistical design and inference in high-dimensional investigations. Methods Mol Biol 2009; 553: 181-206.

[10] Page GP, Edwards JW, Gadbury GL, et al. The PowerAtlas: a power and sample size atlas for microarray experimental design and research. BMC Bioinformatics 2006; 7: 84.

[11] Wu Z, Irizarry RA. Preprocessing of oligonucleotide array data. Nat Biotechnol 2004; 22: 656-8; author reply 658.
[12] Khaitovich P, Hellmann I, Enard W, et al. Parallel patterns of evolution in the genomes and transcriptomes of humans and chimpanzees. Science 2005; 309: 1850-4.

[13] Edgar R, Domrachev M, Lash AE. Gene Expression Omnibus: NCBI gene expression and hybridization array data repository. Nucleic Acids Res 2002; 30(1): 207-10.

[14] Rocke DM, Lorenzato S. A two component model for measurement error in analytical chemistry. Technometrics 1995; 37: 176-84.

[15] Zhang B, Kirov S, Snoddy J. WebGestalt: an integrated system for exploring gene sets in various biological contexts. Nucleic Acids Res 2005; 33: 741-8.

[16] Zhang B, Schmoyer D, Kirov S, Snoddy J. GOTree Machine (GOTM): a web-based platform for interpreting sets of interesting genes using Gene Ontology hierarchies. BMC Bioinformatics 2004; 5: 16.

[17] Rozen S, Skaletsky H. Primer3 on the WWW for general users and for biologist programmers. Methods Mol Biol 2000; 132: 365-86.

[18] Vandesompele J, De Preter K, Pattyn F, et al. Accurate normalization of real-time quantitative RT-PCR data by geometric averaging of multiple internal control genes. Genome Biol 2002; 3: 0034.1-11.

[19] U.S. department of health and human services: 2004 annual report of the U.S. organ procurement and transplantation network and the scientific registry of transplant recipients: Transplant data 19942003.

[20] Terasaki PI, Cecka JM, Gjertson DW, Takemoto S. High survival rates of kidney transplants from spousal and living unrelated donors. N Engl J Med 1995; 333: 333-6.

[21] Jassem W, Koo DD, Cerundolo L, Rela M, Heaton ND, Fuggle SV. Cadaveric versus living donor livers: differences in inflammatory markers after transplantation. Transplantation 2003; 76:1599-603.

[22] Boom H, Mallat MJ, de Fijter JW, Zwinderman AH, Paul LC. Delayed graft function influences renal function, but not survival. Kidney Int 2000; 58: 859-66.

[23] Koning OH, Ploeg RJ, van Bockel JH, et al. Risk factors for delayed graft function in cadaveric kidney transplantation: a prospective study of renal function and graft survival after preservation with University of Wisconsin solution in multi-organ donors. European Multicenter Study Group. Transplantation 1997; 63: $1620-8$.

[24] Offermann G. What is a reasonably short cold ischemia time in kidney transplantation? Transplant Proc 1998; 30: 4291-3.

[25] Audibert G, Charpentier C, Seguin-Devaux C, et al. Improvement of donor myocardial function after treatment of autonomic storm during brain death. Transplantation 2006; 82: 1031-6.

[26] van Der Hoeven JA, Ter Horst GJ, Molema G, et al. Effects of brain death and hemodynamic status on function and immunological activation of the potential donor liver in the rat. Ann Surg 2000; 232: 804-13.

[27] Pérez López S, Vazquez Moreno N, Escudero Augusto D, et al. A molecular approach to apoptosis in the human heart during brain death. Transplantation 2008; 86: 977-82.

[28] Boutros T, Nantel A, Emadali A, et al. The MAP kinase phosphatase-1 MKP-1/DUSP1 is a regulator of human liver response to transplantation. Am J Transplant 2008; 8: 2558-68.

[29] Defamie V, Cursio R, Le Brigand K, et al. Gene expression profiling of human liver transplants identifies an early transcriptional signature associated with initial poor graft function. Am J Transplant 2008; 8: 1221-36. 